\title{
Design of a Test Section to Analyze Magneto-Convection Effects in WCLL Blankets
}

\author{
C. Koehly, * L. Bühler, and C. Mistrangelo \\ Karlsruhe Institute of Technology, Karlsruhe, Germany
}

\begin{abstract}
The water cooled lead lithium (WCLL) blanket is one of the European concepts for a Demonstration nuclear fusion reactor (DEMO). The spatial distribution of the water cooling pipes inside the liquid metal blanket breeder zone is a critical issue since efficient heat removal from the liquid metal has to be ensured, avoiding local hot spots in the fluid or in blanket walls. Convective motion, driven by density gradients due to volumetric heat sources in the liquid breeder and heat removal by cooling pipes, is affected by magnetohydrodynamic interactions of the electrically conducting lead lithium with the external magnetic field. For the recent complex design of the DEMO WCLL blanket, prediction of the liquid metal flow is quite difficult. Preliminary numerical and experimental studies are necessary to determine the flow distribution resulting from the combined interaction of electromagnetic forces, buoyancy, and pressure. A test section based on a simplified model geometry supported by preliminary numerical simulations has been designed for experiments in the MEKKA laboratory at the Karlsruhe Institute of Technology and is presented in this paper.
\end{abstract}

Keywords - Water cooled lead lithium blanket, fusion, magnetohydrodynamic, magneto convection.

\section{INTRODUCTION}

One of the European liquid metal blanket concepts considered for a Demonstration nuclear fusion reactor (DEMO) is the water-cooled lead lithium (WCLL) breeding blanket. ${ }^{1}$ The liquid metal alloy lead lithium $(\mathrm{PbLi})$ is used as breeder, neutron multiplier, and heat carrier. Pressurized water flows through a large number of cooling pipes immersed in the liquid metal and in the blanket structure and cools the walls and breeding zone (see Fig. 1).

The distribution of the water-cooling pipes in the breeding zone is a critical issue. Efficient heat removal from the liquid metal has to be ensured, avoiding occurrence of local hot spots. In order to gain the first insight into the flow distribution resulting from the combined interaction of electromagnetic forces, buoyancy, and pressure, preliminary numerical and experimental studies of

*E mail: christina.koehly@kit.edu buoyant magnetohydrodynamic (MHD) flows are foreseen. To be relevant for applications in WCLL blankets, these investigations should cover liquid metal heat transfer and magneto-convective flow in a geometry with internal obstacles simulating the cooling pipes in the blanket.

A simplified model geometry for studying buoyant MHD flows in cavities with internal obstacles will be used for these studies. The test section consists of a liquid metal-filled box, in which a heated pipe simulates neutron heating while a cooled pipe removes the heat from the liquid metal. The magneto-convective flow is driven by buoyancy when heat is exchanged between the liquid metal and the cooling and heating pipes. Unfortunately, volumetric neutron heating as in a blanket is technically impossible in the experiment. However, we are confident that the major magnetoconvective phenomena can be reproduced well with the designed test section. The dimensions of the box have been chosen such that the experiment fits into the gap of 


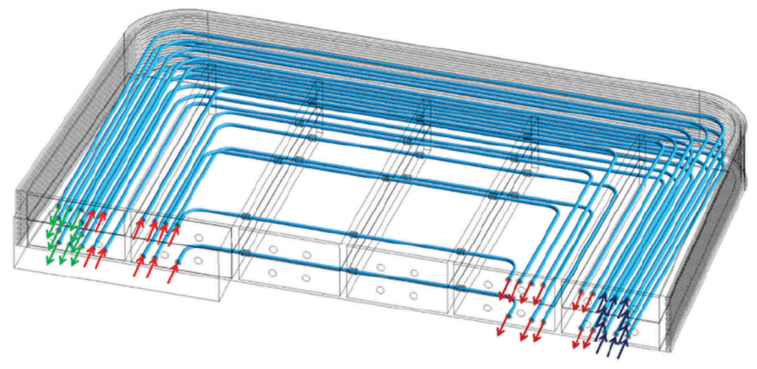

Fig. 1. WCLL blanket design [ENEA (Ref. 2)]: equator ial section in a blanket module with cooling pipes.

the dipole magnet available in the MEKKA laboratory at the Karlsruhe Institute of Technology (KIT), where a quite uniform magnetic field is achieved in a domain of $800 \times 480 \times 168 \mathrm{~mm}^{3}$ (Ref. 3). The design of the experimental test section is presented in this paper. Results of the experiments and numerical simulations will improve the understanding of magneto-convective flows and help to optimize the design of the cooling system in the breeding zone and to demonstrate the feasibility of the proposed WCLL DEMO design.

\section{PRELIMINARY NUMERICAL SIMULATIONS}

Preliminary numerical simulations have been performed for buoyant flow in a model geometry with volumetric heating. The heat was removed by a single cooling pipe in a rectangular channel. The simulations predict the liquid metal flow and heat transfer in strong magnetic fields, where thin layers develop along magnetic field lines, tangential to the cooling pipe., ${ }^{4,5}$

In order to support the design of the present experiment with some theoretical data, numerical simulations have been performed in which the driving temperature difference between the heated pipe and the cooled pipe has been set to $\Delta T=T 2-T 1=40 \mathrm{~K}$. The strength of the magnetic field is measured in terms of the nondimensional Hartmann number:

$$
H a=B L \sqrt{ } \sigma / \rho v,
$$

where

$$
B=\text { magnetic field }
$$

$L=$ half-length of the box in field direction

$\sigma, \rho, v=$ electric conductivity, density, and kinematic viscosity of the fluid.

All walls are electrically insulating and adiabatic.

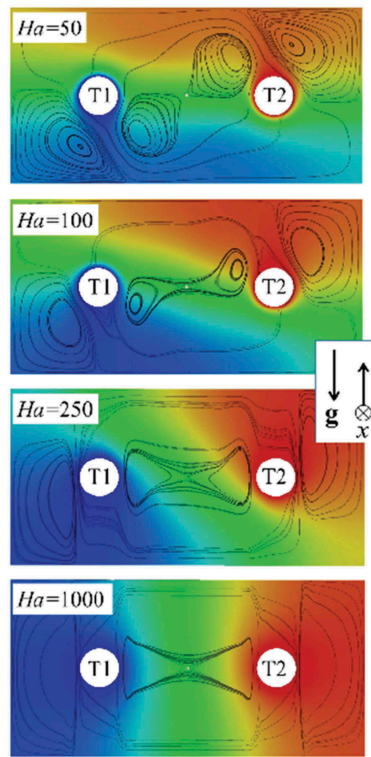

(a)
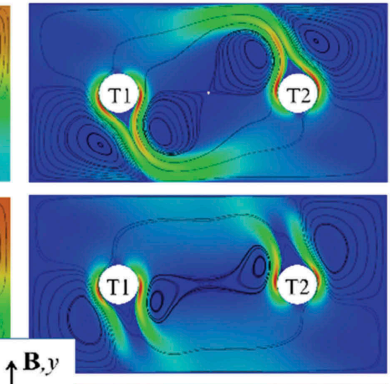
$\underset{\& \longrightarrow}{\mathrm{B}, y}$
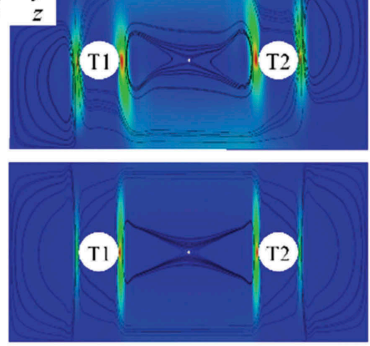

(b)
Fig. 2. (a) Contours of temperature and (b) magnitude of velocity on a cross section of the geometry. Streamlines in black. Magneto convective flow for $\Delta T$ $T 2 \quad T 1 \quad 40 \mathrm{~K}$.

Some results of numerical simulations are shown in Fig. 2 for different strengths of the magnetic fields, i.e., for different Hartmann numbers. The images in Fig. 2a show contours of fluid temperature. Flow streamlines indicate that there are convective cells present in the center between the two pipes and the maximum velocity occurs near the walls of the tubes. For a small $\mathrm{Ha}=50$, the liquid metal flow and the convective heat transport are strong enough to influence the temperature distribution. Hot fluid is transported toward the cold pipe and vice versa. When increasing the magnetic field, the intensity of the convective motion reduces significantly, and temperature and velocity distributions become symmetric with respect to the vertical midplane. For $H a=1000$, the maximum velocity is localized in thin layers parallel to the magnetic field and tangent to the pipes. The fluid velocity in the rest of the box remains quite small. It should be mentioned that in the numerical simulations it is assumed that the box is long enough that the flow reaches fully developed thermal and hydrodynamic conditions normal to the considered plane. Consequently, no axial electric potential difference builds up, and electric currents induced in the liquid metal circulate exclusively in the axial direction. Since the experiment will have finite axial length, it is expected that three-dimensional (3-D) phenomena with measurable potential differences will occur near the end walls. Corresponding 3-D numerical simulations are ongoing. 
According to these numerical results, suitable positions for measuring temperature are along magnetic field lines. Interesting velocity data are expected in parallel layers tangent to the pipes, and electric potential differences might be seen only on the end walls of the box, where electric currents have to close in thin viscous layers.

\section{DESIGN AND INSTRUMENTATION}

\section{III.A. Test Section}

Figure 3 shows two transparent views of the experimental WCLL test section design in an isometric view and top view. It consists of a rectangular box made of Peek plates. Peek can be used for higher temperatures, and it is compatible with the model fluid GaInSn. Peek plates are designed to form a leak-tight rectangular box. They are assembled with screws as well as a thin layer of silicon flat seal and a one-component adhesive based on silicon. All

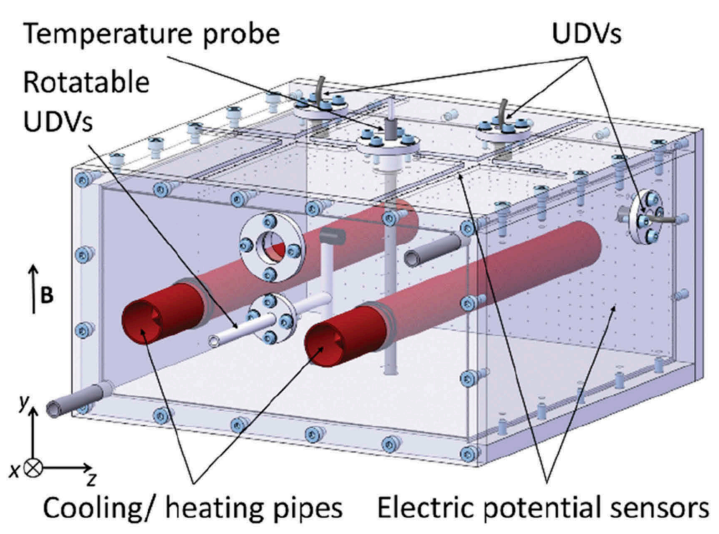

(a)

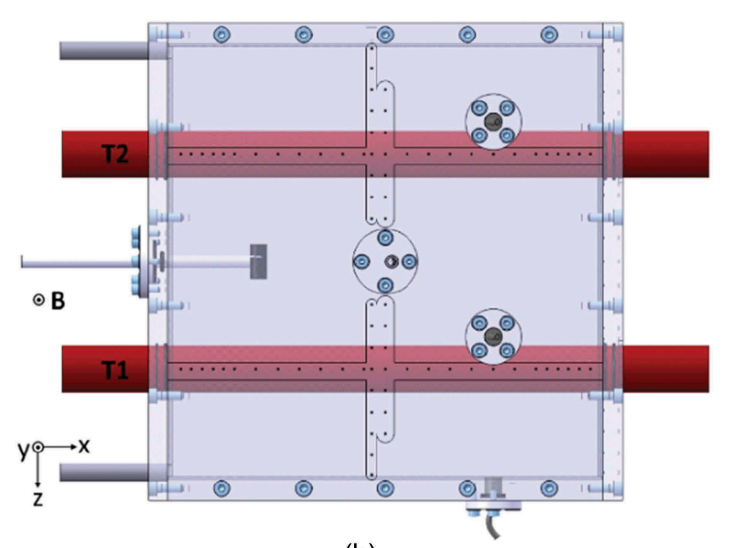

(b)

Fig. 3. Design of the experimental test section in (a) isometric view and (b) top view. lead-ins into the box, for the thermocouple probe or ultrasound Doppler velocimetry (UDV) sensors, are closed with circular caps sealed by O-rings and fixed with screws. For draining and venting of the mock-up, two small access pipes are attached to the front plate.

All parts of the box have been fabricated in the workshops of KIT. Figure 4 shows the fabricated and assembled parts before instrumentation.

Two parallel copper pipes are placed inside the box and sealed with two O-rings at the front plate and back plate. The outer surface of the pipes has been coated with a $2-\mu \mathrm{m}$ thin silicon carbide layer (SiC-Silcor). The coating is thin enough for good thermal conductivity but provides sufficient protection against corrosion attack. The pipes are kept at a constant temperature difference $\Delta T=T 2-T 1$ during the experiments by internal water cooling or heating. For that reason, each pipe is connected to a temperature-controlled water circuit, which allows establishing the desired temperature difference in the box. Copper has been used as pipe material due to its good thermal conductivity. The pipes are supplied with copper cores for achieving as good as possible isothermal conditions (Fig. 5).

The copper cores have been designed to have eight channels circumferentially distributed, where the tempered water cools or heats the pipes. There are another eight small grooves were thermocouples are placed at different axial and circumferential positions. Thereby, the temperature can be monitored during the experiments near the inlet and the outlet and at the center of the pipes.

\section{III.B. Instrumentation of the Test Section}

The aim of the experiments is measuring simultaneously several physical quantities such as liquid metal velocity,

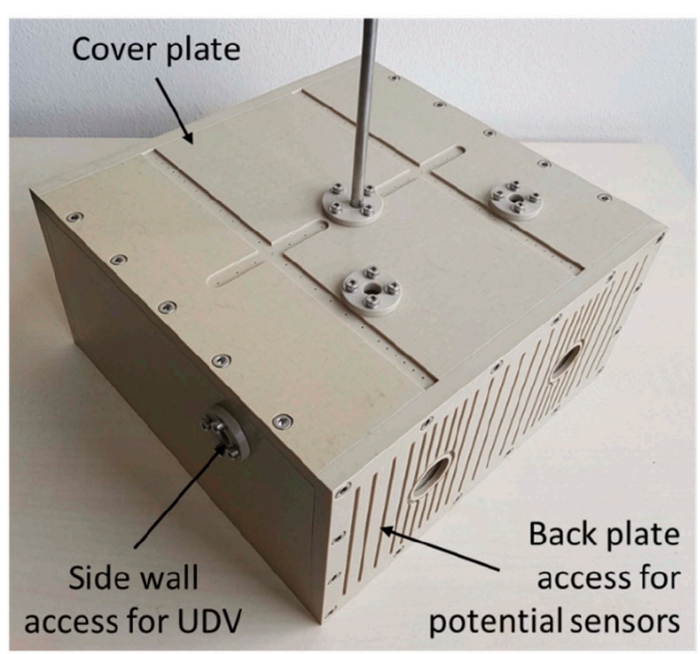

Fig. 4. WCLL mock up without instrumentation. 


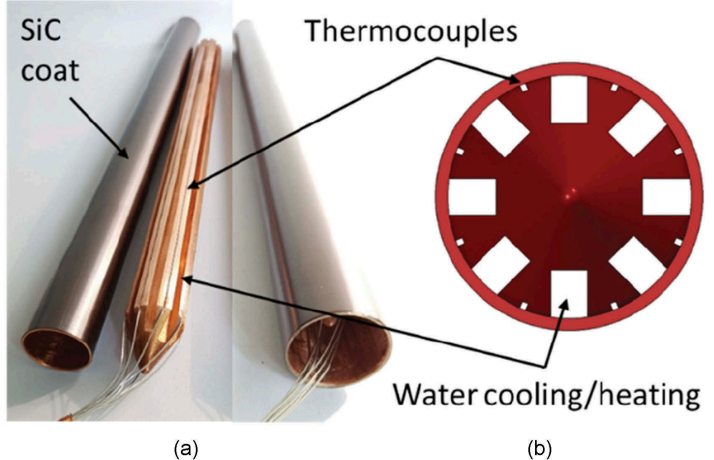

Fig. 5. (a) Copper pipe and core with inserted thermo couples and (b) front view of the design.

electric potential on the surface of the box, and temperature distribution at numerous points within the fluid. For measuring the vertical temperature distribution in the center of the test section, a temperature probe has been fabricated, which has 11 thermocouples equidistantly distributed over the height. Installation of the probe is possible via an O-ringsealed flange on the upper wall. Figure 6 shows the temperature probe with thermocouples assembled in the test section.

In addition, the temperature on the walls of the box is monitored in more than 50 positions in order to obtain an overview of the temperature distribution and to estimate the amount of transferred heat. The thermocouples used for the experiment should be able to give precise results also in high magnetic fields. Standard thermocouples such as type $\mathrm{K}$ are known to be problematic in magnetic environments. Manufacturer experience with thermocouples in high magnetic fields suggests that thermocouples of type $\mathrm{T}(\mathrm{Cu}-\mathrm{CuNi})$ are most suitable for accurate results while offering similar sensitivity to that of type K. For that reason, all thermocouples are of type T. Numerical calculations show that small temperature differences have to be resolved during the experiment, which requires a very precise data acquisition system. This is achieved by measuring all temperatures with respect to

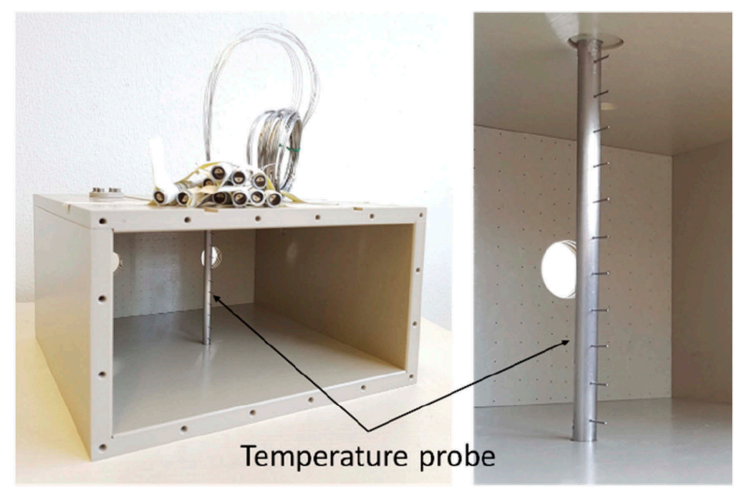

Fig. 6. Temperature probe inside the mock up.

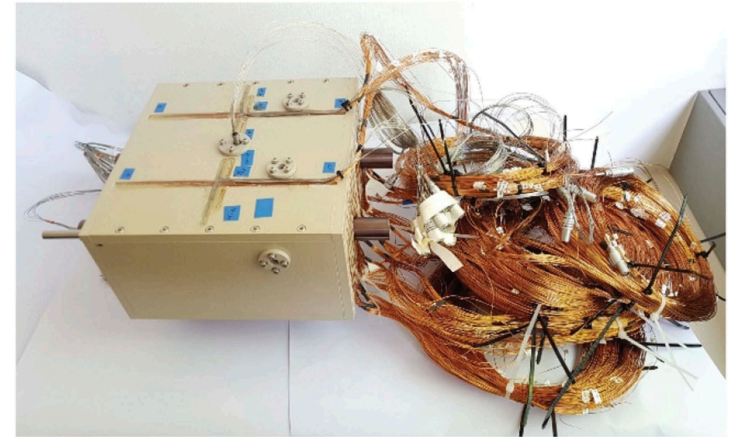

Fig. 7. Instrumented WCLL mock up with copper cables and thermocouples.

a multichannel high-precision type $\mathrm{T}$ reference ice point, for all incoming signals.

In MHD flows, electric potential is a property that can be measured with high precision for comparison with twodimensional and 3-D numerical results. Electric potential will be measured on the fluid-solid interface by a large number of copper electrodes that penetrate specific walls of the test section. The highest flow-induced values of potential are expected at the vertical end walls. Therefore, one of those walls is instrumented with a dense population of electrodes (see Fig. 7). The cables will be connected to a multichannel multiplexer and a multichannel nanovoltmeter.

Preliminary numerical simulations show that the highest velocities occur in thin layers tangent to the heating/cooling pipes. It is planned to record velocity data from those layers by using UDV (Ref. 6). A principal sketch showing the position of the sensors and the propagation zones of the ultrasonic pulses is displayed in Fig. 8. Two sensors will give information (velocity component in the direction of pulse propagation) about the flow in the tangent layers and one in the cores.

For measuring velocity along other directions, a movable probe with two UDV sensors is foreseen. The probe is mounted on a rotatable rod and can be turned on in the $y-z$ plane (see Fig. 9). It is centered in the middle of the box. The rotatable shaft is guided through the front plate, and it can be rotated during the experiment from outside for scanning a large portion of the $y-z$ plane.

\section{PRELIMINARY EXPERIMENTS WITH WATER}

Before the test section is filled with liquid metal, first experiments will be performed using water as a test fluid. These preliminary tests are foreseen to show full functionality of the instrumentation, and they will have already given results for the hydrodynamic limit, when no magnetic field is present. Moreover, unlike liquid 


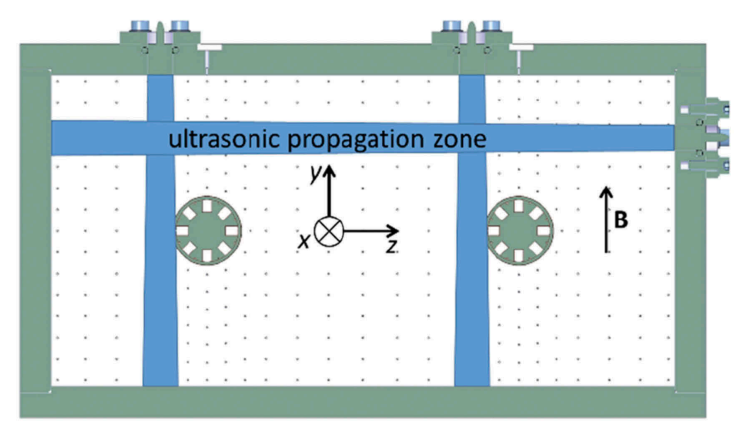

Fig. 8. Sketch showing positions of UDV sensors on the walls and propagation zones of ultrasonic pulses, in which the beam aligned component of velocity can be detected.

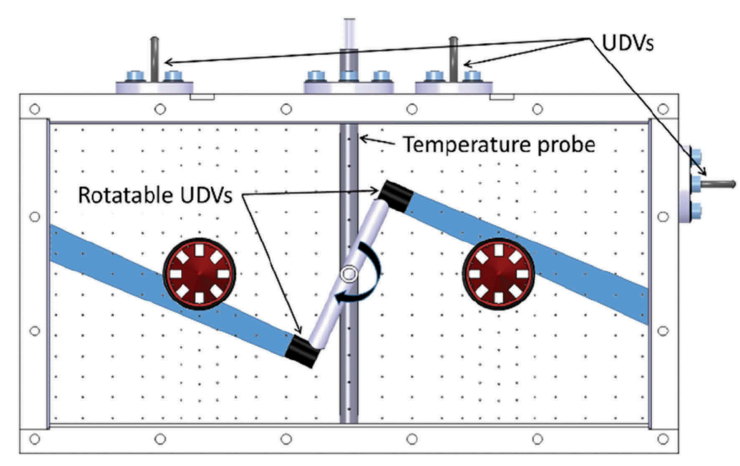

Fig. 9. Instrumentation of the test section: thermocouples at probe and inside the copper pipes, electric potential sensors on the end wall, UDV sensors fixed at walls or on rotatable probe.

metals, water is transparent, which allows optical access and application of particle image velocimetry for flow visualization. For this purpose, end walls and one side wall that are already instrumented for liquid metal experiments (see Fig. 7) are replaced by transparent material. The test section for these preliminary experiments is shown in Fig. 10.

A sketch of the optical setup is shown in Fig. 11. A laser light sheet illuminates the $y-z$ plane, while the movement of seeding particles with the flow in this plane is observed by a camera along the $x$ direction.

\section{CONCLUSIONS}

In order to investigate experimentally magnetoconvective liquid metal flows for WCLL blankets, a test section with a simplified model geometry has been designed and manufactured, supported by preliminary numerical simulations. The test section is instrumented for simultaneously measuring temperature, potential, and velocity distribution. It is foreseen to perform experiments for various values of the

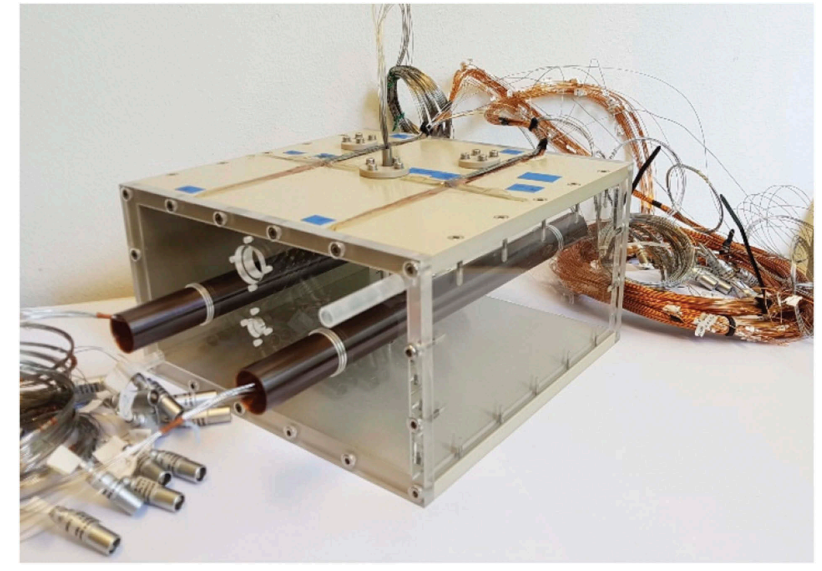

Fig. 10. WCLL mock up for preliminary experiments with water: front, side, and back walls made of Plexiglas.

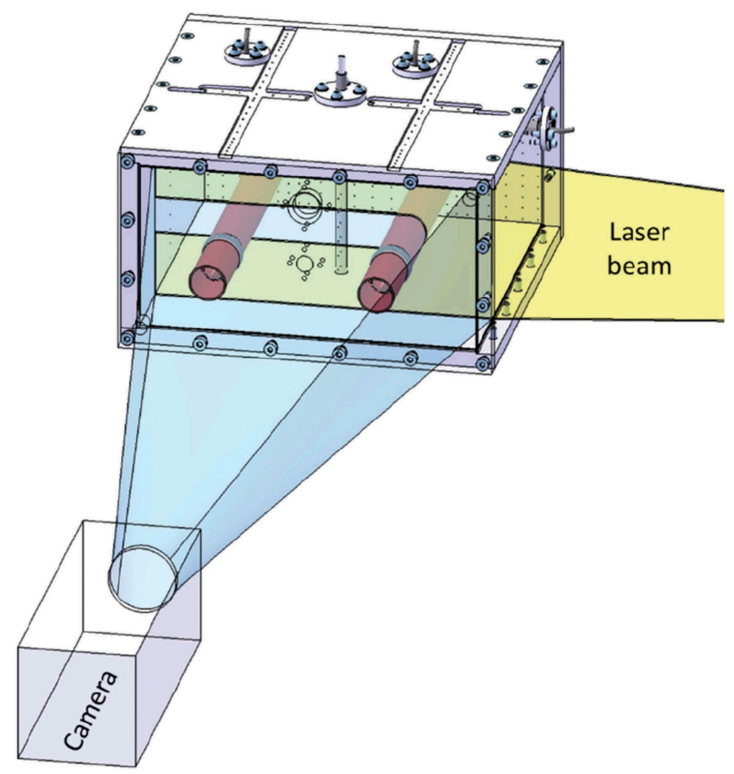

Fig. 11. Arrangement of test section, laser beam, and cam era for preliminary hydrodynamic experiments with water.

applied magnetic field and driving temperature differences, i.e., for various Hartmann and Grashof numbers, respectively. The WCLL model geometry consists of a box made of Peek plates, where two parallel copper pipes simulate the cooling tubes and volumetric heating in the blanket module. They generate the nonuniform temperature and density distribution that drive the buoyant flow under the influence of gravity.

First experiments will be performed with water instead of liquid metal. This will allow us to test instrumentation for temperature measurements and to obtain, with laser optical methods, velocity results in the hydrodynamic limit. In a second step, the test section will be filled with liquid metal GaInSn and exposed to magnetic fields for final magneto-convection experiments. 


\section{Acknowledgments}

This work has been carried out within the framework of the EUROfusion Consortium and has received funding from the Euratom research and training program 20142018 under grant agreement number 633053 .

\section{References}

1. F. ROMANELLI et al., "Fusion Electricity: A Roadmap to the Realisation of Fusion Energy," European Fusion Development Agreement (2012).
2. A. TASSONE et al., "Recent Progress in the WCLL Breeding Blanket Design for the DEMO Fusion Reactor," IEEE Trans. Plasma Sci., 46, 5, 1446 (2018).

3. L. BARLEON, K. J. MACK, and R. STIEGLITZ, "The MEKKA Facility A Flexible Tool to Investigate MHD Flow Phenomena," Forschungszentrum Karlsruhe (1996).

4. C. MISTRANGELO and L. BÜHLER, "Magneto Convective Flows in Rectangular Ducts with Internal Cylindrical Obstacles," presented at 17th Annual Mtg. MHD Days 2016, Göttingen, Germany, November 30 December 2, 2016.

5. L. BÜHLER and C. MISTRANGELO, "MHD Flow and Heat Transfer in Model Geometries for WCLL Blankets," Fusion Eng. Des., 122, 919 (2017); https://doi.org/10.1016/j. fusengdes.2017.01.014.

6. D. BRITO et al., "Ultrasonic Doppler Velocimetry in Liquid Gallium,” Exp. Fluids, 31, 653 (2001); https://doi.org/10. $1007 / \mathrm{s} 003480100312$. 
Karlsruher Institut für Technologie

\section{Repository KITopen}

Dies ist ein Postprint/begutachtetes Manuskript.

Empfohlene Zitierung:

Koehly, C.; Bühler, L.; Mistrangelo, C.

Design of a Test Section to Analyze Magneto-Convection Effects in WCLL Blankets. 2019. Fusion science and technology, 75

doi: $\underline{10.5445 / I R / 1000095709}$

Zitierung der Originalveröffentlichung:

Koehly, C.; Bühler, L.; Mistrangelo, C.

Design of a Test Section to Analyze Magneto-Convection Effects in WCLL Blankets. 2019. Fusion science and technology, 75, 1-6

doi:10.1080/15361055.2019.1607705 